BULLETIN OF THE

AMERICAN MATHEMATICAL SOCIETY

Volume 79, Number 2, March 1973

\title{
A FUNDAMENTAL SOLUTION FOR A SUBELLIPTIC OPERATOR ${ }^{1}$
}

\author{
BY G. B. FOLLAND
}

Communicated by E. M. Stein, August 15, 1972

1. Introduction. Let $\mathscr{L}: C^{\infty}(M) \rightarrow C^{\infty}(M)$ be a formally selfadjoint differential operator of order 2 on the Riemannian manifold $M . \mathscr{L}$ is said to be subelliptic of order $\varepsilon(0<\varepsilon<1)$ at $x \in M$ if there exist a neighborhood $V$ of $x$ and a constant $c>0$ such that for all $u \in C_{0}^{\infty}(V)$,

$$
\|u\|_{\varepsilon}^{2} \leqq c\left(|(\mathscr{L} u, u)|+\|u\|^{2}\right),
$$

where $\|u\|$ is the $L^{2}$ norm and $\|u\|_{\varepsilon}$ is the Sobolev norm of order $\varepsilon$. According to a fundamental theorem of Kohn and Nirenberg [3], subelliptic operators are hypoelliptic and satisfy the $a$ priori estimates

$$
\|u\|_{s+2 \varepsilon}^{2} \leqq c_{s}\left(\|\mathscr{L} u\|_{s}^{2}+\|u\|^{2}\right), \quad u \in C_{0}^{\infty}(V),
$$

for each $s \geqq 0$.

In this note we shall display an operator on a Euclidean space which is subelliptic of order $\frac{1}{2}$ at each point and construct an explicit integral operator which inverts it.

2. Construction of the operator. Let $N$ be the nilpotent Lie group whose underlying manifold is $\boldsymbol{C}^{n} \times \boldsymbol{R}$ with coordinates $\left(z_{1}, \ldots, z_{n}, t\right)=(z, t)$ and whose group law is

$$
(z, t)\left(z^{\prime}, t^{\prime}\right)=\left(z+z^{\prime}, t+t^{\prime}+2 \operatorname{Im} z \cdot z^{\prime}\right)
$$

where $z \cdot z^{\prime}=\sum_{1}^{n} z_{j} \bar{z}_{j}^{\prime}$. Letting $z=x+i y$, then, $x_{1}, \ldots, x_{n}, y_{1}, \ldots, y_{n}, t$ are real coordinates on $N$. We set

$$
\begin{aligned}
X_{j} & =\frac{\partial}{\partial x_{j}}+2 y_{j} \frac{\partial}{\partial t}, & Y_{j} & =\frac{\partial}{\partial y_{j}}-2 x_{j} \frac{\partial}{\partial t}, \\
\frac{\partial}{\partial z_{j}} & =\frac{1}{2}\left(\frac{\partial}{\partial x_{j}}-i \frac{\partial}{\partial y_{j}}\right), & \frac{\partial}{\partial \bar{z}_{j}} & =\frac{1}{2}\left(\frac{\partial}{\partial x_{j}}+i \frac{\partial}{\partial y_{j}}\right), \\
Z_{j} & =\frac{1}{2}\left(X_{j}-i Y_{j}\right), & \bar{Z}_{j} & =\frac{1}{2}\left(X_{j}+i Y_{j}\right) .
\end{aligned}
$$

The following proposition is easily verified.

AMS(MOS) subject classifications (1970). Primary 35C05, 35H05, 43A80; Secondary 35B45, 44A25.

Key words and phrases. Subelliptic operators, fundamental solutions, singular integrals, analysis on nilpotent groups, regularity of solutions.

${ }^{1}$ This research was partially supported by a Sloan Foundation Grant. 
Lemma 1. $X_{1}, \ldots, X_{n}, Y_{1}, \ldots, Y_{n}, T$ are a basis for the Lie algebra of $N$.

We impose the left-invariant metric on $N$ which makes this basis orthonormal at each point and note that the induced volume element is Lebesgue measure, which we denote by $d(z, t)$.

THEOREM 1. The operator

$$
\mathscr{L}=\sum_{1}^{n}\left[-\frac{\partial^{2}}{\partial z_{j} \partial \bar{z}_{j}}-\left|z_{j}\right|^{2} \frac{\partial^{2}}{\partial t^{2}}+i \frac{\partial}{\partial t}\left(z_{j} \frac{\partial}{\partial z_{j}}-\bar{z}_{j} \frac{\partial}{\partial \bar{z}_{j}}\right)\right]
$$

is left-invariant and is subelliptic of order $\frac{1}{2}$ at each $x \in N$.

Proof. One easily sees that $\mathscr{L}=-\frac{1}{2} \sum_{1}^{n}\left(Z_{j} \bar{Z}_{j}+\bar{Z}_{j} Z_{j}\right)$, which by Lemma 1 implies left-invariance. Moreover, since $Z_{j}$ is the formal adjoint of $-\bar{Z}_{j}$, we have

$$
(\mathscr{L} u, u)=\frac{1}{2} \sum_{1}^{n}\left(\left\|\bar{Z}_{j} u\right\|^{2}+\left\|Z_{j} u\right\|^{2}\right), \quad u \in C_{0}^{\infty}(N) .
$$

We invoke the following special case of a theorem of Kohn [2] and Radkevič [5]:

LEMMA 2. Let $V$ be a compact set in a Riemannian manifold $M$, and let $L_{1}, \ldots, L_{N}$ be complex vector fields on $M$ whose linear span is closed under complex conjugation and such that $\left\{L_{j}\right\}_{1}^{N} \cup\left\{\left[L_{j}, L_{k}\right]\right\}_{j, k=1}^{N}$ spans the tangent space at each $x \in V$. Then there exists $c>0$ such that for all $u \in C_{0}^{\infty}(V)$,

$$
\|u\|_{1 / 2}^{2} \leqq c\left(\sum_{1}^{N}\left\|L_{j} u\right\|^{2}+\|u\|^{2}\right) .
$$

The hypotheses of Lemma 2 are satisfied if we take the $L_{j}$ 's to be $Z_{1}, \ldots, Z_{n}, \bar{Z}_{1}, \ldots, \bar{Z}_{n}$, since $\left[\bar{Z}_{j}, Z_{j}\right]=2 i T$. Hence (3) implies (1), and the theorem is proved.

REMARK. $N$ is the nilpotent part in the Iwasawa decomposition of the holomorphic automorphism group of the Siegel domain

$$
\left\{\zeta \in C^{n+1}: \sum_{1}^{n}\left|\zeta_{j}\right|^{2}-\operatorname{Im} \zeta_{n+1}<0\right\}
$$

and it may be identified with the boundary of the domain via the correspondence $(z, t) \leftrightarrow\left(z_{1}, \ldots, z_{n}, t+i \sum_{1}^{n}\left|z_{j}\right|^{2}\right)$. Under this identification, $-2 \sum_{1}^{n} Z_{j} \bar{Z}_{j}$ is just the "tangential complex Laplacian" $\square_{b}$ of J. J. Kohn (cf. $[1])$, and hence $\mathscr{L}=\frac{1}{4}\left(\square_{b}+\square_{b}\right)$. Also, note that when $n=1$, the operator $\bar{Z}=(\partial / \partial \bar{z})-i z(\partial / \partial t)$ is the "unsolvable" operator of H. Lewy [4].

3. Construction of the fundamental solution. Following Stein [6], we 
introduce the group $\left\{\delta_{r}: 0<r<\infty\right\}$ of dilations on $N$ defined by $\delta_{r}(z, t)=$ $\left(r z, r^{2} t\right)$, which satisfy the distributive law $\delta_{r}\left((z, t)\left(z^{\prime}, t^{\prime}\right)\right)=\left(\delta_{r}(z, t)\right)\left(\delta_{r}\left(z^{\prime}, t^{\prime}\right)\right)$, and we define the norm function $\rho(z, t)=\left(|z|^{4}+t^{2}\right)^{1 / 4}$ (where $|z|^{2}=z \cdot z$ ), which satisfies $\rho\left(\delta_{r}(z, t)\right)=r \rho(z, t)$. By analogy with the fact that $|x|^{2-m}$ is (a constant multiple of) the fundamental solution of the Laplacian on $\boldsymbol{R}^{\boldsymbol{m}}$ with source at 0 , we now prove

THEOREM 2. $c_{n} \rho^{-2 n}$ is a fundamental solution for $\mathscr{L}$ with source at 0 , where

$$
c_{n}=\left[n(n+2) \int_{N}|z|^{2}\left(\rho(z, t)^{4}+1\right)^{-(n+4) / 2} d(z, t)\right]-1 .
$$

In other words, for any $u \in C_{0}^{\infty}(N),\left(\mathscr{L} u, c_{n} \rho^{-2 n}\right)=u(0)$.

Proof. Given $\varepsilon>0$, let $\rho_{\varepsilon}=\left(\rho^{4}+\varepsilon^{4}\right)^{1 / 4}$; a simple calculation then shows that

where

$$
\left(\mathscr{L} \rho_{\varepsilon}^{-2 n}\right)(z, t)=\varepsilon^{-2 n-2} \phi\left(\delta_{1 / \varepsilon}(z, t)\right)
$$

$$
\phi(z, t)=n(n+2)|z|^{2}\left(\rho(z, t)^{4}+1\right)^{-(n+4) / 2} .
$$

From the fact that $\varepsilon^{-2 n-2} \int_{N} \phi \circ \delta_{1 / \varepsilon}=\int_{N} \phi=c_{n}^{-1}<\infty$ and the fact that $\delta_{1 / \varepsilon}(V) \rightarrow N$ as $\varepsilon \rightarrow 0$ for any neighborhood $V$ of 0 , it now follows easily that for any $u \in C_{0}^{\infty}(N)$,

$$
\left(\mathscr{L} u, c_{n} \rho^{-2 n}\right)=\lim _{\varepsilon \rightarrow 0}\left(\mathscr{L} u, c_{n} \rho_{\varepsilon}^{-2 n}\right)=\lim _{\varepsilon \rightarrow 0}\left(u, c_{n} \mathscr{L} \rho_{\varepsilon}^{-2 n}\right)=u(0),
$$

and the theorem is proved.

Since $\mathscr{L}$ is left-invariant, we deduce immediately

COROLlary 1. If $f \in C_{0}^{\infty}(N)$, then the function $u=f *\left(c_{n} \rho^{-2 n}\right)$ is a solution of $\mathscr{L} u=f$, where $*$ denotes convolution on the group $N$.

The hypothesis on $f$ can be relaxed considerably, of course. For example, the convolution integral will converge absolutely provided that $f \in L^{n+1-\varepsilon} \cap L^{n+1+\varepsilon}$ for some $\varepsilon>0$.

4. Applications. We shall now prove a precise regularity theorem for $\mathscr{L}$ by means of the theory of singular integrals on nilpotent groups (cf. [6] and the references given there). A singular integral kernel on $N$ is a function of the form $\Omega \rho^{-2 n-2}$ where $\Omega$ is a smooth function on $N-\{0\}$ satisfying $\Omega\left(\delta_{r}(z, t)\right)=\Omega(z, t)$ for all $r>0$ and $\int_{a<\rho(z, t)<A} \Omega(z, t) d(z, t)=0$ for all $0<a<A<\infty$. If $\psi$ is a singular integral kernel, the operator $f \rightarrow f * \psi$, the convolution integral being defined in a suitable principal-value sense, enjoys the same basic properties as Calderon-Zygmund operators on $\boldsymbol{R}^{\boldsymbol{m}}$ : it is bounded on $L^{p}, 1<p<\infty$, and is weak type $(1,1)$. 
THEOREM 3. Let $u=f *\left(c_{n} \rho^{-2 n}\right)$ as in Corollary 1. Then the operators taking $f$ to $X_{j} X_{k} u, Y_{j} Y_{k} u, X_{j} Y_{k} u, Y_{j} X_{k} u(j, k=1, \ldots, n)$ and $T u$ (but not $X_{j} T u, Y_{j} T u$, or $T^{2} u$ ) are bounded on $L^{p}, 1<p<\infty$, and are weak type $(1,1)$.

Proof. Computations similar to those in the proof of Theorem 2 show that the distribution derivatives $T \rho^{-2 n}, X_{j} Y_{k} \rho^{-2 n}, Y_{j} X_{k} \rho^{-2 n}$, and, for $j \neq k, X_{j} X_{k} \rho^{-2 n}$ and $Y_{j} Y_{k} \rho^{-2 n}$ are singular integral kernels, and the distribution derivatives $X_{j}^{2} \rho^{-2 n}$ and $Y_{j}^{2} \rho^{-2 n}$ are singular integral kernels plus multiples of the Dirac $\delta$-function at 0 . The theorem now follows immediately from the definition of $u$ and the left-invariance of $X_{j}, Y_{j}$, and $T$.

By the same reasoning, of course, we can estimate higher derivatives of $u$ in terms of appropriate derivatives of $f$ by shifting some of the derivatives onto $f$ in the convolution defining $u$. This yields a very precise interpretation of the estimates (2) as well as their extension to $L^{p}, p \neq 2$ : Passage from $f$ to $u$ gains one derivative in the $T$ direction and two derivatives in all directions orthogonal to $T$.

We hope to elaborate on these ideas in a future publication.

\section{REFERENCES}

1. G. B. Folland and J. J. Kohn, The Neumann problem for the Cauchy-Riemann complex, Ann. of Math. Studies, no. 75, Princeton Univ. Press, Princeton, N.J., 1972.

2. J. J. Kohn, Pseudo-differential operators and non-elliptic problems, Pseudo-Differential Operators (C.I.M.E., Stresa, 1968), Edizioni Cremonese, Rome, 1969, pp. 157-165. MR 41 \# 3972.

3. J. J. Kohn and L. Nirenberg, Non-coercive boundary value problems, Comm. Pure Appl. Math. 18 (1965), 443-492. MR 31 \#6041.

4. $\mathrm{H}$. Lewy, An example of a smooth linear partial differential equation without solution, Ann. of Math. (2) 66 (1957), 155-158. MR 19, 551.

5. E. V. Radkevič, Hypoelliptic operators with multiple characteristics, Mat. Sb. 79 (121) (1969), 193-216 = Math. USSR Sb. 8 (1969), 181-205. MR 41 \# 5763.

6. E. M. Stein, Some problems in harmonic analysis suggested by symmetric spaces and semi-simple groups, Proc. Internat. Congress Math. (Nice, 1970), vol. 1, Gauthier-Villars, Paris, 1971, pp. 173-189.

Courant Institute of Mathematical Sciences, New York University, New York, NEW YORK 10012. 